\title{
Letter to the Editor $\$$
}

\section{Industry and public bealth Tackling the infant formula industries}

Madam

Arne Oshaug has summarised the penetration of nutrition science conferences by problematic sectors of the food, drink and allied industries ${ }^{(1)}$. Here we summarise how the baby formula manufacturers have infiltrated the midwifery and allied health professions, and outline an example of good practice.

Formula feed companies fund midwifery and healthbased conferences, advertise and provide sponsorship within relevant journals, and promote their products via the literature disseminated by trade union organisations.

In the UK, where we work, representatives from formula feed companies have had unregulated access to health professionals. They circulate 'free' products through these contacts, together with marketing disguised as professional information, and in such ways covertly recruit professionals as marketing assistants.

These and other formula food industry tactics undermine the International Code of Marketing of Breast-milk Substitutes $^{(2)}$. This code was developed as a minimum international standard to ensure the proper use and regulation of breast-milk substitutes and to curb unethical marketing practices, false and or misleading promotion, and advertisements by industry of their products ${ }^{(3)}$. Compliance with this code is a key requirement of the Global Strategy for Infant and Young Child Feeding ${ }^{(4)}$, as well as the WHO/UNICEF Baby-Friendly Hospital Initiative ${ }^{(5)}$. Arne Oshaug's commentary argues for reform of relationships with industries, as does an editorial in the same volume ${ }^{(6)}$. Clear, transparent guidelines are recommended. We agree.

We report that fulfilment of the WHO code is being achieved as part of the community Baby-Friendly Initiative (BFI) award within two primary care trusts in the North-West of England. This model provides an open and transparent framework and guidelines to address how relevant health professionals should engage with industry.

The BFI requires health premises and practitioners to provide consistent advice, information and support on infant feeding based on the best available evidence. The award is designed to support the infrastructure of health or hospital facilities, such as through the creation of policies, protocols, training, information networks and evaluation mechanisms. The BFI accreditation status is issued to facilities deemed to have reached the minimum externally auditable standards.
Current national BFI guidance offers little insight as to how the international marketing code may be achieved. In NHS North Lancashire and NHS Blackpool, links with formula industry representatives is sustained, but without their having access to health premises. This has been achieved through the creation of an Infant Feeding Information Team (IFIT). The team is the sole point of contact for formula industry representatives. In this way, all breast- and bottle-feeding information is assessed, to ensure compliance with BFI best practice standards. No information supplied via the health facilities and professionals may contain advertisements. The team is also developing guidelines to support the BFI Breastfeeding Policy and to disseminate key infant feeding information.

Representatives from the formula companies are invited to present information about their products to the team. They are also required to provide evidence which can validate and uphold their claims, as well as provide timely updates when changes are to be made to their products. Members from various professional groups, such as health visitors, public health leaders, midwives and voluntary organisations, attend team meetings.

The team appraises infant feeding information, including empirical evidence, and leaflets and booklets produced. An IFIT report is produced every month and electronically disseminated. The team also identifies and replaces accessories and tools previously used by professionals that display logos of these formula companies (such as on diary covers, leaflets, pens and lanyards). Formula companies have immense advertising budgets, and the eradication of advertisements is therefore an ongoing challenge for the team. The team has also submitted topic suggestions, in order for systematic reviews and guidance to be undertaken, to the National Institute for Health and Clinical Excellence.

To date, the IFIT team has received regional as well as national recognition. A representative from the group was invited to speak at an event for national infant feeding coordinators by the Department of Health, and the creation of a regional (North West) IFIT team is currently under discussion.

The team aims to offer a framework of transparency, regulation and accountability within which the breastmilk substitute industry can engage with health professional groups, and it challenges and appraises evidence presented and claims made by these companies. The team also engages with a variety of activities concerning infant feeding, and promotes and supports breast-feeding. We believe IFIT is a model of good practice, and has the potential to be adopted at national level. 


\section{Gill Thomson \\ Fiona Dykes}

MAINN Unit, School of Public Health \& Clinical Sciences

University of Central Lancashire, Preston PR1 2HE, UK

Email: GThomson@uclan.ac.uk

Andy Bilson (Professor)

School of Social Work

University of Central Lancashire

Preston PR1 2HE, UK

Jane Putsey

Mary Whitmore

NHS Blackpool/NHS North, Lancashire, UK

Sally Dickens

NHS North Lancashire, Moor Lane

Lancaster, LA1 1QD, UK

doi:10.1017/S1368980009992886

\section{References}

1. Oshaug A (2009) What is the food and drink industry doing in nutrition conferences? (Invited commentary). Public Health Nutr 12, 1019-1020.

2. World Health Organization (1981) International Code of Marketing of Breastmilk Substitutes. Geneva: WHO.

3. Aguayo VM, Ross JS, Kanon S et al. (2003) Monitoring compliance with the International Code of Marketing of Breastmilk Substitutes in West Africa: multisite cross sectional survey in Togo and Burkina Faso. BMJ 326, 127-130.

4. World Health Organization (2003) Global Strategy for Infant and Young Child Feeding. Geneva: WHO.

5. UNICEF (2009) WHO/UNICEF Baby Friendly Initiative. http://www.babyfriendly.org.uk (accessed July 2009).

6. Margetts B (2009) Time to agree guidelines and apply an ethical framework for public health nutrition (Editorial). Public Health Nutr 12, 885-886. 